\title{
ALTERAÇÕES MICROESTRUTURAIS NO ESMALTE RELACIONADAS AO CLAREAMENTO DENTAL
}

\section{MODIFICATIONS OF ENAMEL RELATED TO DENTAL BLEACHING}

André Rodrigo Justino da Silva

Universidade Federal de Campina Grande - UFCG

\begin{abstract}
In view of the adverse effects that tooth bleaching can cause to the teeth, the present literature review was carried out on the changes in the microstructural levels that the enamel suffers from whitening. The search was made in the databases Scielo and Google scholar for articles published only in the last ten years, from the keywords hydrogen peroxide, dental bleaching, dental enamel, adverse effects and microscopy electron scanning. From the fifteen articles selected it was observed that all the authors show that there are alterations in the enamel structure due to the bleaching agents, which reduce the microhardness of the fabric and increase its roughness, besides causing erosion, pores, among other losses minerals. In addition, the use of some complementary techniques in bleaching, such as prior acid conditioning, the use of LED activation and the application of subsequent neutral fluoride increase damage to the dental structure, while others, such as the presence of calcium in the gel composition bleaching, do not demonstrate interfere in the mineral structure of the element. Moreover, the use of the casein phosphopeptide compound/amorphous calcium phosphate appears to be promising when associated with bleaching peroxide, and further studies on its applicability are required. Thus, it is concluded that, despite being conservative, dental bleaching has the potential to promote mineral losses on the surface of the tooth enamel.
\end{abstract}

Keywords: Tooth Bleaching. Dental Enamel.ToothDemineralization. Microscopy, Electron

\section{Resumo}

Diante dos efeitos adversos que o clareamento dental pode causar aos dentes realizou-se a presente revisão de literatura sobre as alterações a níveis microestruturais que o esmalte sofre decorrente desse procedimento. Foi feita a busca nas bases de dados Scielo e Google acadêmico por artigos publicados somente nos últimos dez anos, a partir das palavras-chave hydrogenperoxide, dental bleaching, dental enamel, adverse effects e microscopyelectronscanning. A partir dos quinze artigos selecionados observou-se que todos os autores mostram que existem alterações na estrutura do esmalte decorrente dos agentes clareadores, que diminuem a microdureza do tecido e aumentam a sua rugosidade, além de causar surgimento de erosões, poros, dentre outras perdas minerais. Além disso, o uso de algumas técnicas complementares no clareamento, como o condicionamento ácido prévio, o uso de ativação por LED e a aplicação de flúor neutro subseqüente aumentam os danos à estrutura dental, enquanto outros, como a presença de Cálcio na composição do gel clareador, não demonstram interferir na estrutura mineral do elemento. Por outro lado, o uso do composto de fosfopeptídeos de caseína/fosfato de cálcio amorfo aparenta ser promissor quando associado ao peróxido clareador, sendo necessários mais estudos sobre essa aplicabilidade. Desse modo conclui-se que, apesar de ser conservador, o clareamento dental desempenha potencial para promover perdas minerais na superfície do esmalte dental.

Palavras chave: Clareamento dental. Esmalte dentário. Desmineralização do dente. Microscopia eletrônica. 
A cor que os dentes apresentam constitui o fator mais importante no estabelecimento do equilíbrio estético do sorriso, apesar de ser apenas um dentre os fatores que colaboram para isso, já que a alteração de cor é a desarmonia mais rapidamente percebida e notada por quem observa. As abordagens de tratamento para esses casos dependem principalmente da origem da alteração, sendo assim de suma importância a capacidade de diagnóstico refinado do profissional para conseguir indicar o melhor tratamento para o paciente. Com isso o clareamento dental se mostra como uma

abordagem eficaz na reversão de alterações de cor $^{1}$.

Atualmente, os agentes clareadores disponíveis para o tratamento de dentes vitais são vários, com diferentes concentrações tanto para a técnica de clareamento em consultório quanto à técnica caseira, desse modo possibilitando aos profissionais alternativas para a escolha do mais indicado para cada caso ${ }^{2}$.

Os agentes clareadores utilizados nas técnicas de clareamento do esmalte dental são peróxidos que tem como mecanismo de ação a oxidação de compostos orgânicos alcançada pela sua facilidade de penetração nas estruturas dentais, por possuir baixo peso molecular. As moléculas do pigmento responsáveis pela alteração de cor são quebradas em versões reduzidas e como resultado dessa reação há a liberação de oxigênio, que penetra nos túbulos dentinários e age convertendo os compostos pigmentados em compostos mais claros ${ }^{2,3,4}$.

Porém, a ação do agente clareador tem um limite chamado de ponto de saturação, a partir do qual ele começa a agir quebrando moléculas que possuem composição semelhante às pigmentadas, mas que apresentam papel estrutural para o dente, desse modo alterando a microestrutura do esmalte e podendo aumentar a fragilidade do elemento dental ${ }^{1,4}$.

Há registros do aparecimento de alterações na estrutura dental e em tecidos circunvizinhos com a realização de tratamento clareador, além do aumento da hipersensibilidade durante e após o clareamento, sendo esta referida como o efeito adverso mais frequente. Assim, com esse achado se cria a hipótese de que os géis utilizados no clareamento dental tratamento são capazes de alterar a morfologia, estrutura ou composição molecular dos elementos dentais com os quais entram em contato ${ }^{5}$. Assim, frente aos efeitos adversos que os agentes clareadores podem causar à estrutura do dente, realizou-se a presente revisão de literatura como objetivo de identificar as alterações a níveis microestruturais que acontecem no esmalte dentário decorrente do clareamento dental.

\section{Metodologia}

Foi realizada busca nas bases de dados Scielo e Google Acadêmico para obtenção dos artigos para a revisão. A pesquisa foi feita com o uso das palavras-chave hydrogen peroxide, dental bleaching, dental enamel, adverse effects e microscopyelectronscanning. Foram inclusos artigos em inglês e português publicados somente entre os anos 2009 a 2019 e artigos que fizeram uso de dentes de origem bovina como objeto de pesquisa, já que os dentes bovinos se mostram como compatíveis substitutos de dentes humanos. Foram excluídos deste estudo os trabalhos de conclusão de curso, teses e dissertações.

\section{Resultados e discussões}

Inicialmente foram selecionados 23 trabalhos, porém após leitura dos resumos foram filtrados 15 que se encaixavam nos critérios de inclusão para a revisão. Todos os artigos relataram alterações na estrutura do esmalte decorrentes dos agentes usados no clareamento dental, sendo eles peróxidos de diferentes composições e concentrações, como está descrito na tabela 01 com respectivo autor do artigo e seu ano de publicação.

Ao comparar agentes clareadores com e sem a presença de cálcio na composição observou-se que, no protocolo utilizado em consultório não há diferença na microdureza entre os agentes, nem entre os tempos de avaliação. Entretanto, entre os tratamentos caseiros houve redução significativa da microdureza após o fim dos tratamentos, porém entre os agentes clareadores com e sem cálcio não houve diferença. Assim, a presença de cálcio não interfere na microdureza do esmalte dental tanto imediatamente quanto após sete dias da conclusão do tratamento ${ }^{6}$.

Ao avaliar a rugosidade superficial do esmalte dental de dentes bovinos após o contato durante 14 dias com peróxido de hidrogênio a 6,5\% por 30 minutos diários; peróxido de hidrogênio a 7,5\% por uma hora diária e peróxido de hidrogênio a 7,7\% por 30 minutos diários, os autores identificaram que o uso do peróxido de hidrogênio com menor concentração não 
Tabela 1. Informações sobre os trabalhos utilizados na revisão.

\begin{tabular}{|c|c|c|}
\hline AUTORES & ANO & $\begin{array}{l}\text { AGENTE(S) CLAREADOR(ES) } \\
\text { UTILIZADO(S) }\end{array}$ \\
\hline KUTUK; ERGIN; CAKIR; GURGAN. & 2019 & Peróxido de hidrogênio a $38 \%$. \\
\hline GRUBER; COLLI; MUNIZ; OTTONI; BORBA. & 2018 & $\begin{array}{l}\text { Peróxido de hidrogênio a } 35 \% \text { e } \\
\text { Peróxido de carbamida a } 10 \% \text {. }\end{array}$ \\
\hline $\begin{array}{l}\text { OLIVEIRA; CARVALHO; OTTONI; SILVA; VAN } \\
\text { DE SANDE. }\end{array}$ & 2018 & Peróxido de hidrogênio a $35 \%$. \\
\hline VIEIRA; ANDRADE; OLIVEIRA; SILVA. & 2017 & $\begin{array}{l}\text { Peróxido de hidrogênio a 6,5\%, 7,5\% e } \\
7,7 \% \text {. }\end{array}$ \\
\hline ARAÚJO; ARAÚJO; AGUIAR. & 2016 & Peróxido de carbamida a $16 \%$. \\
\hline $\begin{array}{l}\text { SANTANA; NAVARRO; LIMA; FERNANDES; } \\
\text { AMARAL; FRANÇA; TURSSI; BASTING. }\end{array}$ & 2016 & Peróxido de hidrogênio a 37,5\% \\
\hline $\begin{array}{l}\text { SOARES; BOMBONATTI; ALENCAR; } \\
\text { CONSOLMAGNO; HONÓRIO; MONDELLI. }\end{array}$ & 2016 & $\begin{array}{l}\text { Peróxido de hidrogênio a } 35 \%, 25 \% \text {, } \\
15 \%, 10 \% \text {. }\end{array}$ \\
\hline $\begin{array}{l}\text { BERGER; SOARES; MARTIN; AMBROSANO; } \\
\text { TABCHOURY; GIANNINI. }\end{array}$ & 2014 & $\begin{array}{l}\text { Peróxido de hidrogênio a } 10 \%, 35 \% \text { e } \\
50 \%\end{array}$ \\
\hline $\begin{array}{l}\text { HORNING; GOMES; BITENCOURT; RUIZ; } \\
\text { REIS; GOMES. }\end{array}$ & 2013 & Peróxido de hidrogênio a $35 \%$ e $6 \%$. \\
\hline $\begin{array}{l}\text { SOARES; RIBEIRO; SACONO; LOGUÉRCIO; } \\
\text { HEBLING; COSTA. }\end{array}$ & 2013 & $\begin{array}{l}\text { Peróxido de carbamida a } 16 \% \text { e } \\
\text { Peróxido de carbamida a } 10 \% .\end{array}$ \\
\hline AYRES; BERGER; ANDRÉ; GIANNINI. & 2012 & $\begin{array}{l}\text { Peróxido de carbamida a } 16 \% \text { e } \\
\text { Peróxido de hidrogênio a } 35 \% \text { e } 37,5 \% \text {. }\end{array}$ \\
\hline $\begin{array}{l}\text { CARDOSO; ODA; JUNIOR; FREITAS; FILHO; } \\
\text { VASCONCELLOS. }\end{array}$ & 2012 & Peróxido de hidrogênio a 35\%. \\
\hline PACHALY; POZZOBON. & 2012 & Peróxido de carbamida a $10 \%$. \\
\hline PINHE IRO; COSTA; KLAUTAU; CARDOSO. & 2011 & $\begin{array}{l}\text { Peróxido de hidrogênio a } 35 \% \text { e } \\
\text { Peróxido de carbamida a } 16 \% \text {. }\end{array}$ \\
\hline $\begin{array}{l}\text { DUDEA; FLOREA; MIHU; CÂMPEANU; } \\
\text { NICOLA; BENGA. }\end{array}$ & 2009 & Peróxido de carbamida a $15 \%$. \\
\hline
\end{tabular}


provocou alterações nas superfícies dos dentes nas primeiras 24 horas em comparação aos agentes de maior concentração, porém ao final do sétimo dia de clareamento observou- se que a microdureza dos dentes submetidos ao agente de menor concentração foi reduzida mostrando assim que, apesar da sua concentração diminuída, esse também é capaz de provocar modificações na rugosidade superficial do esmalte. O grupo que foi submetido ao clareamento com a concentração $7,5 \%$ por uma hora diária apresentou o maior aumento da rugosidade superficial dentre os dentes clareados, sendo justificado pelos autores pelo movimento potente de radicais livres entre o esmalte e a dentina associado ao maior tempo de tratamento 7 .

É importante salientar que o aumento na rugosidade decorrente de tratamento clareador pode facilitar a adesão de bactérias cariogênicas, bem como de biofilme pigmentado podendo assim comprometer o resultado estético do tratamento ${ }^{8}$, como visto no estudo que utilizou nitrato de prata para confirmar que o elemento dental sofre aumento da permeabilidade às alterações de cor devido às perdas minerais em nível de esmalte decorrentes dos protocolos de clareamento ${ }^{9}$.

Utilizando dentes bovinos associados a diferentes concentrações de peróxido de hidrogênio, ácido fosfórico a $37 \%$ e fontes de luz, autores observaram que $\mathrm{o}$ ph dos agentes clareadores diminuiu do início para o final da aplicação, com exceção do grupo que recebeu o protocolo composto pela concentração de $15 \%$ ativado com luz híbrida. Identificaram também que quanto maior a concentração de peróxido de hidrogênio mais ácido é seu ph. Segundo os autores é improvável que, isoladamente, o ph dos agentes clareadores seja capaz de provocar mudanças morfológicas severas na superfície do esmalte, já que em todos os grupos avaliados o ph do peróxido de hidrogênio se manteve acima do nível considerado crítico para promoção da dissolução do esmalte ${ }^{10}$.

Ao utilizar dois tipos de peróxido de carbamida no esmalte de dentes humanos com a mesma concentração observou-se que o uso desses agentes provoca aumento dos poros, erosões e crateras nas superfícies dos dentes quando comparado ao seu não-uso. Segundo os autores as pequenas modificações observadas na superfície do esmalte podem se relacionar ao início de um processo de desmineralização.
Quanto à relação isolada entre o ph do peróxido de carbamida e a perda de minerais do esmalte, novamente observa-se que não há relação direta entre esses fatores, já que o ph do agente clareador se mostrou neutro ${ }^{11}$.

O uso da técnica de condicionamento com ácido fosfórico a $37 \%$ antes do clareamento aumenta a severidade dos desgastes da superfície, o que torna contra-indicado o uso dessa técnica associada ao procedimento de rotina. Sua indicação é para somente graus severos de pigmentação com a finalidade de facilitar a penetração do gel no esmalte ${ }^{10}$. Segundo os autores utilizar a ativação por fonte de luz ou o condicionamento ácido prévio provoca diminuição do tempo de aplicação do agente clareador, porém não aumenta a efetividade do clareamento nem mesmo altera o número de sessões necessárias para alcançar um resultado satisfatório ${ }^{12}$.

A associação do peróxido de hidrogênio a $37,5 \%$ com o Ozônio para o clareamento do esmalte dental mostra que o uso do gás não reduz as alterações na composição estrutural do esmalte. Mesmo com essa junção, o agente clareador se mostra capaz de alterar o esmalte dental a nível microestrutural ${ }^{13}$. Ao utilizar três concentrações distintas de peróxido de hidrogênio, aplicados sob diferentes técnicas, para avaliar seus efeitos sobre o elemento dental observou-se que o agente clareador promoveu alterações no conteúdo mineral e que após a primeira e segunda aplicação o Cálcio presente no esmalte se ligou fracamente à hidroxiapatita e foi perdido independente da concentração do peróxido ${ }^{14}$. Segundo os autores o aumento da concentração não aumenta a perda mineral.

Ao comparar o uso do peróxido de carbamida com a concentração de $10 \%$ e de $16 \%$ observa-se que a redução da microdureza do esmalte é bem maior com o uso da concentração $16 \%$ principalmente após 14 dias de tratamento. Porém ambas as concentrações causam redução do conteúdo de Cálcio e Fósforo em níveis maiores quando comparados ao seu não-uso. Nas duas concentrações o peróxido foi capaz de criar superfícies irregulares com picos e vales numerosos e profundos, provocando assim uma camada microporosa, sendo ela mais grave ao usar a concentração de $16 \%{ }^{15}$. Assim, uma maior concentração de peróxido de carbamida provoca diminuição da microdureza do esmalte dental de forma maior e mais rápida. 
Ao utilizar protocolos de peróxido de carbamida e de hidrogênio, ambos com diferentes apresentações a redução da microdureza do esmalte se mostram como material-dependente. 0 protocolo caseiro provoca maior diminuição dos valores minerais na microestrutura quando comparado ao protocolo de consultório. O gel clareador Whitegold Home -peróxido de carbamida a 16\%(DentsplySirona- York, Pensilvânia, EUA) tanto sozinho quanto associado ao Whitegold Office -peróxido de hidrogênio a 35\%- (DentsplySironaYork, Pensilvânia, EUA) promoveu redução na dureza superficial do esmalte dental bovino, enquanto que o Pola Office e o Pola High -peróxidos de hidrogênio a $37,5 \%$ e de carbamida a $16 \%$, respectivamente- (SDI LimitedBayswater, Victoria, Austrália) não alteram a sua microdureza ${ }^{16}$.

Testando o efeito da escovação, com diferentes dentifrícios, na rugosidade média da superfície do dente humano exposto à ação do peróxido de carbamida a $10 \%$ observa-se que o fato do esmalte ter sido exposto ao agente clareador não influencia na rugosidade obtida após a ação dos diferentes dentifrícios ${ }^{17}$. Ao realizar polimento de superfícies de esmalte dental humano clareado por peróxido de hidrogênio a $38 \%$ com pasta dentifrícia composta por partículas de apatita observa-se aumento da lisura superficial do esmalte ${ }^{8}$. Assim, pode-se dizer que a associação entre a utilização de fluoretos, a presença da saliva e o uso de medidas adequadas de higiene oral podem favorecer o aumento da microdureza do esmalte durante e após o tratamento clareador ${ }^{6}$.

Ao comparar o protocolo de clareamento de consultório utilizando peróxido de hidrogênio a $35 \%$ com o clareamento caseiro utilizando peróxido de carbamida a $16 \%$ observou-se que em ambas as situações ocorreram alterações de aspecto nãouniforme na superfície do esmalte, sendo elas o aumento da quantidade e profundidade dos poros de desenvolvimento, periquimácias com términos mais profundos e algumas áreas de erosão, além de em algumas regiões ter ocorrido depósitos cristalinos $^{18}$. Desse modo, as alterações em esmalte se mostram proporcionais à duração do tratamento e a concentração do peróxido utilizado. O uso do peróxido de carbamida a $15 \%$ promove alterações nas superfícies de esmalte dentário tanto com seu uso por três horas diárias quanto por oito horas diárias em um período de 14 dias $^{19}$.

A aplicação de Flúor e/ou Cálcio é capaz de alterar a perda da microdureza na fase póstratamento, podendo ocorrer a partir da mistura do gel clareador com agentes dessensibilizantes contendo flúor ou com o creme à base de água

CPP-ACP (CaseinPhosphopeptideAmorphousCalciumPhospate). Com o uso do agente clareador sem a presença de dessensibilizante a perda de microdureza se mostra maior do que quando seu uso é adicionado de n-HAP (nano-hidroxiapatita). Os níveis de Cálcio e Fósforo dos tecidos dentais não se mostram alteráveis com a presença de dessensibilizantes no agente clareador, ao contrário dos níveis de Flúor que aumentam com a presença do produto, desse modo prevenindo sensibilidade e/ou desmineralização durante o tratamento ${ }^{20}$. Outro estudo observou que após aplicação tópica de flúor neutro houve aumento da concentração de Flúor, porém sem evidências de redução de perda mineral ${ }^{21}$.

Fazer uso da CCP-ACP na superfície do esmalte dental com grau de desmineralização melhora significativamente o grau de perda mineral, sendo a eficácia ainda maior quando associada ao flúor. Essa efetividade é bastante observada em lesões de mancha branca, apesar de possuir provável eficiência também para superfícies de esmalte dental clareadas ${ }^{22}$.

Ao comparar o uso do peróxido de hidrogênio a $35 \%$ de modo isolado nas apresentações de gel de clareamento sem cálcio

(WhitenessHpmaxx FGM- Joinville, Santa Catarina, Brasil) e gel de clareamento com cálcio (Whiteness HP Blue Calcium FGM- Joinville, Santa Catarina, Brasil) com o peróxido associado ao dessensibilizante os autores não identificaram diferenças estatisticamente significantes quanto ao grau de redução da dureza superficial do esmalte. Sendo assim, a presença do produto para remineralização não mostra influência na perda mineral ${ }^{23}$.

Com relação ao uso de fonte luminosa associada ao tratamento, observou-se aumento da porosidade do esmalte dental com a aplicação de técnica clareadora usando peróxido de hidrogênio a 35\% com ativação por luz LED (Light EmittingDiodes) quando comparado ao uso isolado do peróxido ${ }^{24}$. 
Estes achados não corroboram com o de um estudo in vitro que verificou que, em dentes bovinos a aplicação de peróxido de hidrogênio a $38 \%$ associado à irradiação do produto com luz LED não promove alterações significativas nos tecidos dentais. Os autores deste estudo associaram a ausência de alterações significativas na superfície do esmalte dental com o potencial de remineralização do esmalte pela saliva in vivo ${ }^{25}$. Assim, ainda é bastante controverso afirmar que o tratamento clareador associado à fotoativação torna a superfície do esmalte dental mais rugosa do que a sua realização sem o uso de fonte de luz.

Os produtos de clareamento causam destruição e/ou desnaturação das proteínas da matriz orgânica dos tecidos dentais com consequente efeito potencialmente prejudiciais sobre as propriedades mecânicas do esmalte e da dentina, a exemplo da redução da dureza e do módulo de elasticidade ${ }^{26}$. Portanto, sugere-se que haja cuidado nos protocolos clínicos utilizados, observando o tempo e a frequência da realização do tratamento, além uma correta indicação desses para que sejam evitadas alterações nas propriedades dos tecidos dentais.

Diante dos dados apresentados conclui-se que: as principais alterações microestruturais que o esmalte dental sofre após uso das técnicas de clareamento são: áreas de erosão, poros, crateras e periquimácias com términos mais profundos. A concentração do agente clareador não tem relação direta com o aumento da rugosidade superficial do tecido, ao contrário do tempo de duração do tratamento que se mostra diretamente proporcional ao grau de perda mineral. As alterações estruturais observadas aumentam a permeabilidade do dente propiciando alterações de cor.

\section{Conclusão}

Desse modo observa-se que, apesar de ser considerado um procedimento conservador, o clareamento dental tem potencial para provocar perdas minerais na superfície do esmalte dental. Contudo, deve-se levar em conta que os resultados encontrados foram observados in vitro, assim é preciso considerar a provável atenuação dessas alterações quando se observa o clareamento in vivo, principalmente levando em conta a ação positiva da saliva.

\section{Referências:}

1. Baratieri LN, Junior SM, Andrada MC, Vieira LCC, Ritter AV, Cardoso AC. Odontologia Restauradora. Fundamentos e Possibilidades. Chile, Livraria Santos Editora Comp. Imp. Ltda., 2001.

2. Mendes $M$, Deps SD, Freitas MIM, Frizzera F, Pimentel MJ, Castro GC. Clareamento dental. RevCientFaesa, Vitória, ES, 13 (1), p. 3742, 2017.

3. Conceição EN. Dentística: saúde e estética. 2. ed. Porto Alegre: Artmed, 2007. 583p

4. Soares FF, Sousa JAC, Maia CC, Fontes CM, Cunha LG, Freitas AP. Clareamento em dentes vitais: uma revisão literária. Rev Saúde.com, v. 4, n. 1, p. 72-84, 2008.

5. Spalding M. Estudo 'in vitro' do aspecto morfológico da supefície do esmalte e alteração na permeabilidade dentária após a clareação [Dissertação Mestrado]. Bauru: Universityof São Paulo, Faculdade de Odontologia de Bauru; 2000.

6. Gruber A, Colli L, Muniz C, Ottoni R, Borba $M$. Efeito do uso de agentes clareadores com cálcio na microdureza do esmalte dental. RFO. 22 (3), $2018 . \quad$ Disponível em: http://www.seer.upf.br/index.php/rfo/article/vie w/7655.

7. Vieira A, Andrade A, Oliveira M, Silva I. Influência dos agentes clareadores de baixa concentração sobre a rugosidade superficial do esmalte bovino. RFO. 21 (3), 2017. Disponível em: http://seer.upf.br/index.php/rfo/article/view/61

77.

8. Freitas ACP, Bott SB, Teixeira FS, Salvadori MCBS, Garonenetto N. PSD and gloss of bleached enamel after different polishing procedures. J Dent Res, 2011.

9. Horning D, Gomes GM, Bittencourt BF, Ruiz LM, Reis A, Gomes OMM. Avaliação da permeabilidade ao esmalte humano exposta a agentes clareadores. Braz J Oral Sci. 12 (2): 114-

118, 2013. Disponível em: http://www.scielo.br/scielo.php?script=sci_artte xt\&pid=S1677-32252013000200009\&Ing=en.

10. Soares AF, Bombonatti JFS, Alencar MS, Consolmagno EC, Honório HM, Mondelli RFL. Influence of $\mathrm{pH}$, bleaching agents, and acid etching on surface wear of bovine enamel. J App. Oral Sci. 24 (1): 24-30, 2016. Disponível em: 
http://www.scielo.br/scielo.php?script=sci_artte xt\&pid=S1678-77572016000100024\&Ing=en.

11. Araújo RP, Araújo DB, Aguiar MC. A comparative study of the effects of two carbamide peroxide bleaching agents on the structure of enamel.RGO, RevGaúch Odontol. 64

(3): 293-298, 2016. Disponível em: http://www.scielo.br/scielo.php?script=sci_artte xt\&pid=S1981-86372016000300293\&lng=en.

12. Mondelli RFL, Almeida CM, Ishikiriama SK, Wang L, Atta MT. Influence of enamel acid etching prior to in office bleaching. J Dent Res. 9 (369), 2011.

13. Santana MSCA, Bridi EC, Navarro RS, Lima CJ, Fernandes $A B$, Amaral FLB, et al . Dental bleaching with ozone: effects on color and enamel microhardness. Acta odontollatinoam. 29

(1): 68-75, 2016. Disponível em: http://www.scielo.org.ar/scielo.php?script=sci_a rttext\&pid=S1852-48342016000100011\&lng=es.

14. Berger SB, Soares LES, Martin AA, Ambrosano GMB, Tabchoury CPM, Giannini M. Effects of various hydrogen peroxide bleaching concentrations and number of applications on enamel. Braz J Oral Sci. 13 (1): 22-27, 2014.

Disponível em:

http://www.scielo.br/scielo.php?script=sci_artte xt\&pid=S1677-32252014000100022\&Ing=en.

15. Soares DG, Ribeiro APD, Sacono NT, Loguércio AD, Hebling J, Costa CAS. Mineral Loss and Morphological Changes in Dental Enamel Induced by a $16 \%$ Carbamide Peroxide Bleaching Gel. Braz Dent J. 24 (5): 517-521, 2013.

Disponível

em:

http://www.scielo.br/scielo.php?script=sci_artte xt\&pid=S0103-64402013000500517\&Ing=en.

16. Ayres APA, Berger $S B$, André CB, Giannini M. Avaliação da microdureza do esmalte dental bovino após técnicas de clareamento caseiro, de consultório e a associação das técnicas com agentes de baixa e alta concentração de peróxidos. RPG. 19 (4): 147-152, 2012. Disponível em:

http://revodonto.bvsalud.org/scielo.php?script=s ci_arttext\&pid=S0104-

56952012000400002\&lng=pt.

17. Pachaly R, Pozzobon RT. Analysis of surface roughness of human enamel exposed to bleaching agent and submitted to brushing. Acta odontollatinoam. 25( 1 ): 59-66, 2012. Disponível em:

http://www.scielo.org.ar/scielo.php?script=sci_a rttext\&pid=S1852-48342012000100010\&Ing=es.
18. Pinheiro HB, Costa KG, Klautau EB, Cardoso PEC. Análise microestrutural do esmalte tratado com peróxido de hidrogênio e carbamida. RGO. 59 (2): 215-220, 2011.

Disponível em:

http://revodonto.bvsalud.org/scielo.php?script=s ci_arttext\&pid=S1981-86372011000200007\&lng=pt.

19. Dudea D, Florea A, Mihu C, Câmpeanu R, Nicola $\mathrm{C}$, Benga $\mathrm{GH}$. The use of scanning electron microscopy in evaluating the effect of a bleaching agent on the enamel surface.Rom J MorpholEmbryol. 50 (3): 435-440, 2009.

20. Kutuk ZB, Ergin E, Cakir FY, Gurgan S. Effects of in-office bleaching agent combined with different desensitizing agents on enamel. J

Appl Oral Sci. 2019. Disponível em: http://www.scielo.br/scielo.php?script=sci_artte xt\&pid=S1678-77572019000100406\&Ing=en.

21. PaesLeme AF, Dalcico R, Tabchoury CPM, Del Bel Cury AA, Rosalen PL, Cury JA. In situ

Effect of Frequent Sucrose Exposure on Enamel Demineralization and on Plaque Composition after APF Application and F Dentifrice Use. J Dent Res. 83: 71-75, 2004. Disponível em http://citeseerx.ist.psu.edu/viewdoc/download? doi=10.1.1.528.5061\&rep= rep1\&type=pdf.

22. Melo LFB, Azevedo TDPL, Melo JF, Lima SMF, Bezerra ACB, Toledo OA. Efeito remineralizador da caseína fosfato de cálcio fosfopeptídeo amorfo em dentes decíduos e permanentes in vivo. RevAssoc Paul CirDent. 69

(3): 302-306, 2015. Disponível em: http://revodonto.bvsalud.org/pdf/apcd/v69n3/a 14v69n3.pdf.

23. Oliveira DR, Carvalho RV, Ottoni R, Silva SBA, Leite FHVS. Influência da adição de agente remineralizante em géis clareadores na microdureza e alteração de cor do esmalte dental. J Oral Investig, Passo Fundo, 7 (2): 7-15, 2018.

24. Cardoso RM, Oda M, Andrade JUNIOR ACC, Freitas LM, Sampaio Filho HR, Vansconcellos BT. A rugosidade do esmalte dental e o tratamento clareador. RPG. 19 (2): 39-45, 2012.

Disponível em:

http://revodonto.bvsalud.org/scielo.php?script=s ci_arttext\&pid=S0104-56952012000200001\&lng=pt. 25. Daniel CP, Soares DGS, Andreeta MRB, Hernandes AC, Hebling J, Costa CAS. Efeitos de diferentes sistemas de clareamento dental sobre a rugosidade e morfologia superficial do esmalte 
e de uma resina composta restauradora.

RevOdontolBras Central. 20 (52): 7-14, 2011.

26. FreireJCP, Nóbrega MTC, Ribeiro ED, Carvalho FG, Andrade AKM, Carlo HL. Efeitos do tratamento clareador sobre a dureza e o módulo de elasticidade dos tecidos dentais: Revisão de literatura. Revista da Faculdade de Odontologia de Lins/Unimep. 26 (2) 83-90, 2016.

\section{Endereço para Correspondência}

Universidade Federal de Campina Grande- UFCG

R. Aprígio Veloso, 882 - Universitário, Campina

Grande - PB

CEP.:58428-830

e-mail: a.rodrigo2010@hotmail.com

Recebido em 31/01/2019

Aprovado em 13/12/2019

Publicado em 30/03/2020 